\title{
Aircraft digital assembly process design technology based on 3D Model
}

\author{
Chen Zhen,Tang Jianjun \\ AVIC Chengdu Aircraft Industrial (Group) Co., Ltd. , Chengdu 610092, China
}

\begin{abstract}
In order to improve the quality, efficiency and cooperativity of aircraft assembly, based on the analysis of the characteristics of aircraft assembly process, a method for assembly process design, simulation and application based on three dimensional (3D) product model was proposed. The basic working process and system structure of the method were introduced, and the 3D digital assembly process planning, design and simulation optimization application mode was explored. This method has been applied in the design of aircraft assembly process, the efficiency and synergy of process design have been greatly improved.
\end{abstract}

\section{Introduction}

During the course of an aircraft manufacturing process, there are lots of tasks, such as blank manufacturing, parts machining, assembly and test, but the assembly is the most important and challenging one, and its workload takes more than half of that of the whole manufacturing[1]. Just as we known, we have basically achieved at the stage of 3D digital design, but in aircraft manufacturing, especially in assembly, digital technology is still in its infancy. This is mainly manifested in two aspects: (1) On the one hand, the data transfer is discontinuous, which is contrary to the single data source principle. In order to carry out effective aircraft assembly process design, enterprises have already configured the basic data supporting environment, including PDM, CATIA, DELMIA, 3DVIA Composer, CAPP, etc.[2], but because of the lack of integration among these systems, data transmission is faulting. Large amounts of data in the assembly process design process need to be manually input and maintained. (2)On the other hand, the design of assembly process is lack of cooperativity and verifying. The design of aircraft assembly process depends largely on the personal experience, the process engineer need a lot of time to digest product drawings and understand the assembly relationship between components. Due to the large number of tooling required for aircraft assembly and the complex aircraft structural system, the assembly process design efficiency is relatively low and the accuracy is difficult to guarantee. In addition, because of lacking of the effective measures of pre-verification, many assembly problems were found only in the production site, such as parts assembly interference, the assembly sequence is not reasonable, etc.
Digital assembly process can evaluate flaws in design prior to manufacturing, improving production quality and reducing manufacturing cost[3]. For this purpose, this paper studies the model-based aircraft digital assembly process design technology, including the aircraft digital assembly process design system architecture, design methods and processes.

\section{Review of the literature}

With the extensive application of digital design and manufacturing technology in the aviation manufacturing industry, the research and development of new aircraft has undergone tremendous changes. For example, in the development process of Boeing 777, Boeing has shortened the development cycle by half and the error rework rate has been reduced by $75 \%$ because of the new technology[4]. In the design of digital assembly process, Sierla $\mathrm{S}$ proposes a new concept in which a digital twin derived from a digital product description will automatically perform assembly planning and orchestrate the production resources in a manufacturing cell[5]. J. Ortegón studied the aircraft assembly system optimization problem based on iDMU[6]. Bai Mingxing[7] studied aircraft 3D assembly process design and on-site visualization technology to enhance the quality of process planning and process design. On the basis of analyzing the characteristics of satellite assembly, Sun Gang proposed a simulation and application method of satellite assembly process design based on 3D model, which effectively improved the quality and efficiency of satellite assembly[8]. Guo Hongjie[9] discussed the main technical approaches to realize the $3 \mathrm{D}$ assembly process design based on the model systematically, and studied the key technology of 3D assembly process design, which

Foundation item : "Made in China 2025" Sichuan Action Fund Plan: Aircraft Mobile Intelligent Final Assembly Line( 40207000120X). 
has a certain reference value for realizing the intelligent assembly process design of aircraft.

With the deepening application and development of digital manufacturing technology, the research and application of aircraft digital assembly process design are developing in the following main directions: (1)The transformation of aircraft assembly process design pattern from two-dimensional to three-dimensional. (2)Process design, simulation, optimization and verification based on 3D model. (3) Visual assembly technology.

\section{Methodology and used tools}

The definition of the assembly process can be structured into three main top-level tasks [10]: create conceptual assembly process, define assembly process and develop detailed assembly process. Aircraft assembly process design based on model is an advanced technology and method of assembly process design, which based on three-dimensional model, taking BOM as the support and the unique data source, by constructing a threedimensional assembly process model containing products, processes and resources (products, processes and resources, PPR), on the basis of this model, the assembly process planning, detailed process design and simulation optimization are completed.

The specific process of $3 \mathrm{D}$ assembly process design based on model is as follows:

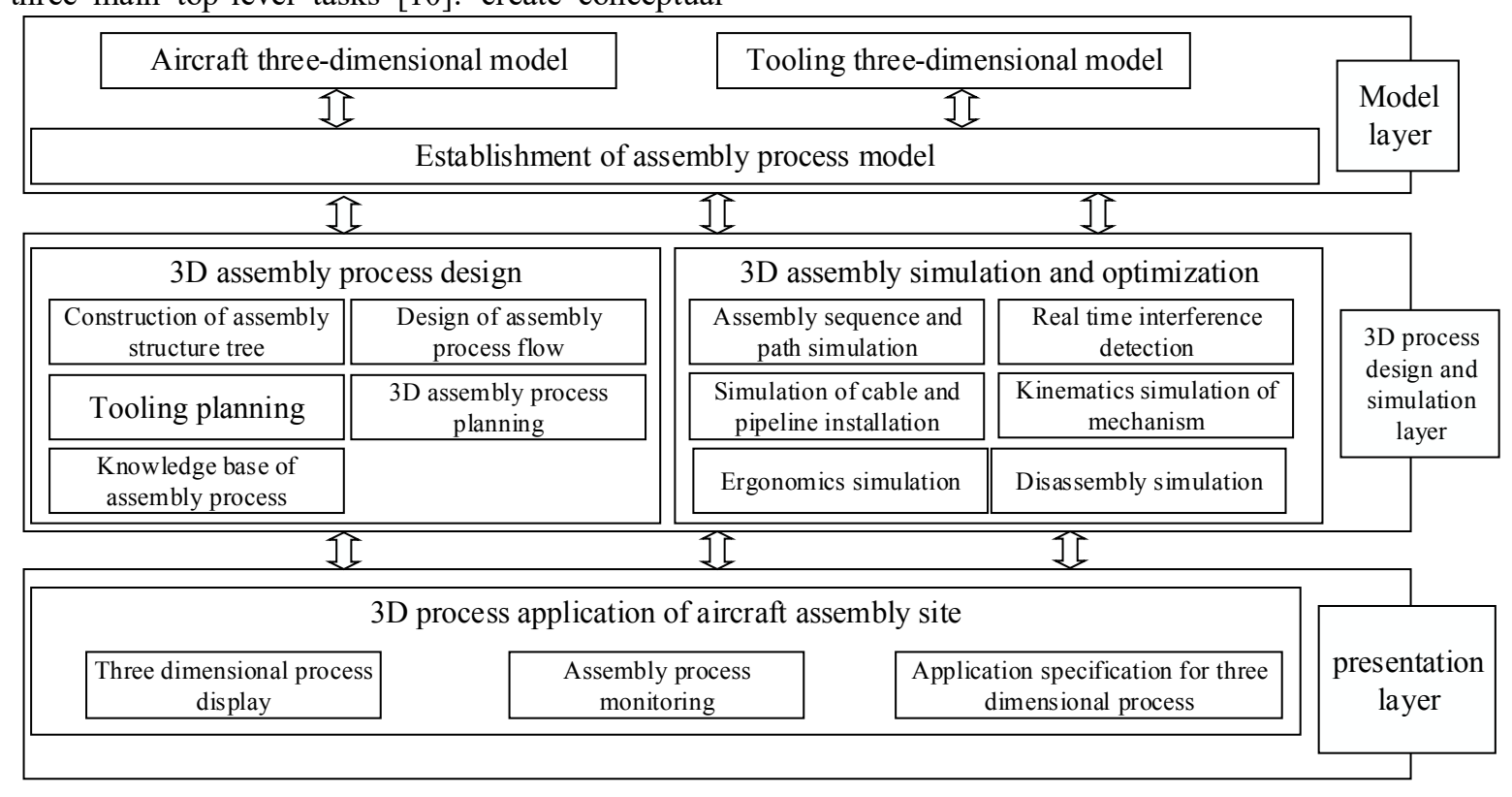

Figure 1. Framework of aircraft assembly process design based model

\subsection{Process planning and design}

The design of aircraft assembly process is carried out from top to bottom, that is to do the overall planning first, and then carry out the detailed design. The general plan is mainly to plan the assembly sequence and transform the EBOM generated by the product into the MBOM required for manufacturing. BOM division is to adjust EBOM appropriately according to the actual needs and process requirements of factories, and form a new BOM structure, including PBOM division and MBOM division.

\subsection{Detailed process planning}

The detailed assembly process design adopts PPR (Process, Product, Resource) document structure. The PPR structure document can directly generate the assembly process model, and create a hierarchical structure of process tree, such as the structure of stations, workstations, and AO (Assembly Order). And PERT diagram is used to plan the relationship between these process nodes, such as serial and parallel. After the assembly process design is completed, MBOM structure can be obtained, which can be provided to ERP or MES.

\subsection{Simulation of assembly process}

Through the three-dimensional lightweight model to carry out assembly process simulation, digital simulation to replace the assembly site physical prototype trial installation process, you can find the process design problems in advance to avoid the process problems left to the production process. The optimized assembly simulation process will guide the assembly site together with the assembly instructions, so that the product can be assembled quickly and accurately, which is beneficial to shorten the assembly cycle and improve the assembly success rate. Assembly simulation includes assembly interference simulation, assembly sequence simulation, ergonomics simulation and so on, and its working process is shown as illustrated. 


\section{Case study}

3DE (3DExperience) is the software platform of digital manufacturing platform developed by Dassault[11]. In the 3DE environment, the process designer uses the manufacturer item definition (MID) module to build the product MBOM; process planning (PP) module is used for product assembly process planning, assembly path planning and assembly simulation, found products design and process planning problems in advance. In the $3 \mathrm{DE}$ platform, in the 3DE platform, the collaborative design of assembly process can be realized. A assembly process collaborative working environment can be set up as follows:

- User A is responsible for Manufacturing Assembly A - User B is responsible for Sub Assembly2

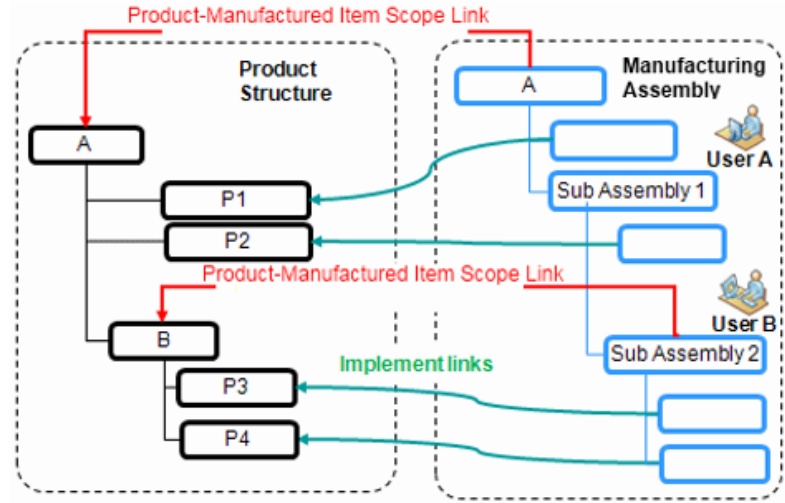

(a)

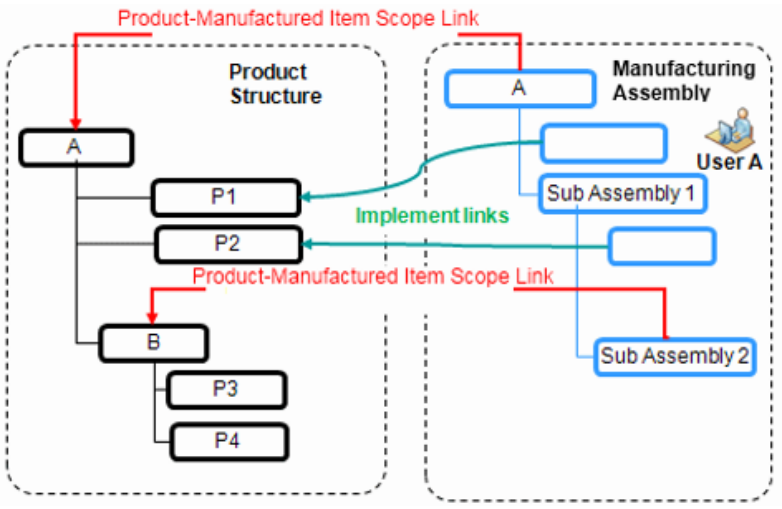

(b)

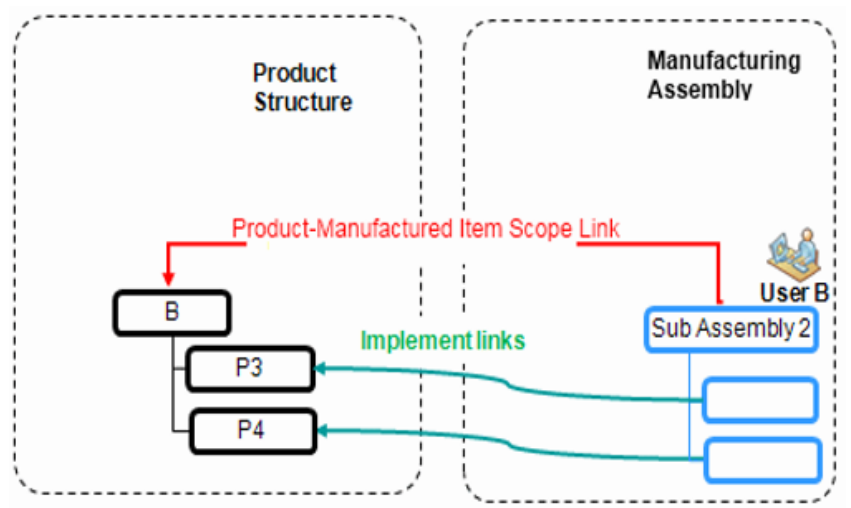

(c)

Figure 2. Cooperative process planning

User A is not concerned about the planning of Product $\mathrm{B}$, the work that remains to be done is clear because the scope of the work is defined and identifiable, user A does not need to know how Product B is manufactured (Fig.2b); User B is not concerned about the planning of Product A, the work that remains to be done is clear because the scope of the work is defined and identifiable (Fig.2c).

(1) Data preparation: Based on the premise and foundation of 3D product digital mold, tooling mold and EBOM, we integrate the 3D model of product and tooling in $3 \mathrm{DE}$ platform and design and plan 3D process with lightweight XML data to ensure the efficiency of assembly process design and simulation work.

(2) Assembly process planning: In the 3DE platform, we use an assembler assistant to implement the rapid construction of MBOM and check the product distribution state through the BI tool, as shown in Figure 3. Drag and drop the EBOM product node from the tree to the top left Manufacturing Assembly tile in the image below. An implement link is created and the product node now has status Assigned directly, with a light blue square. 

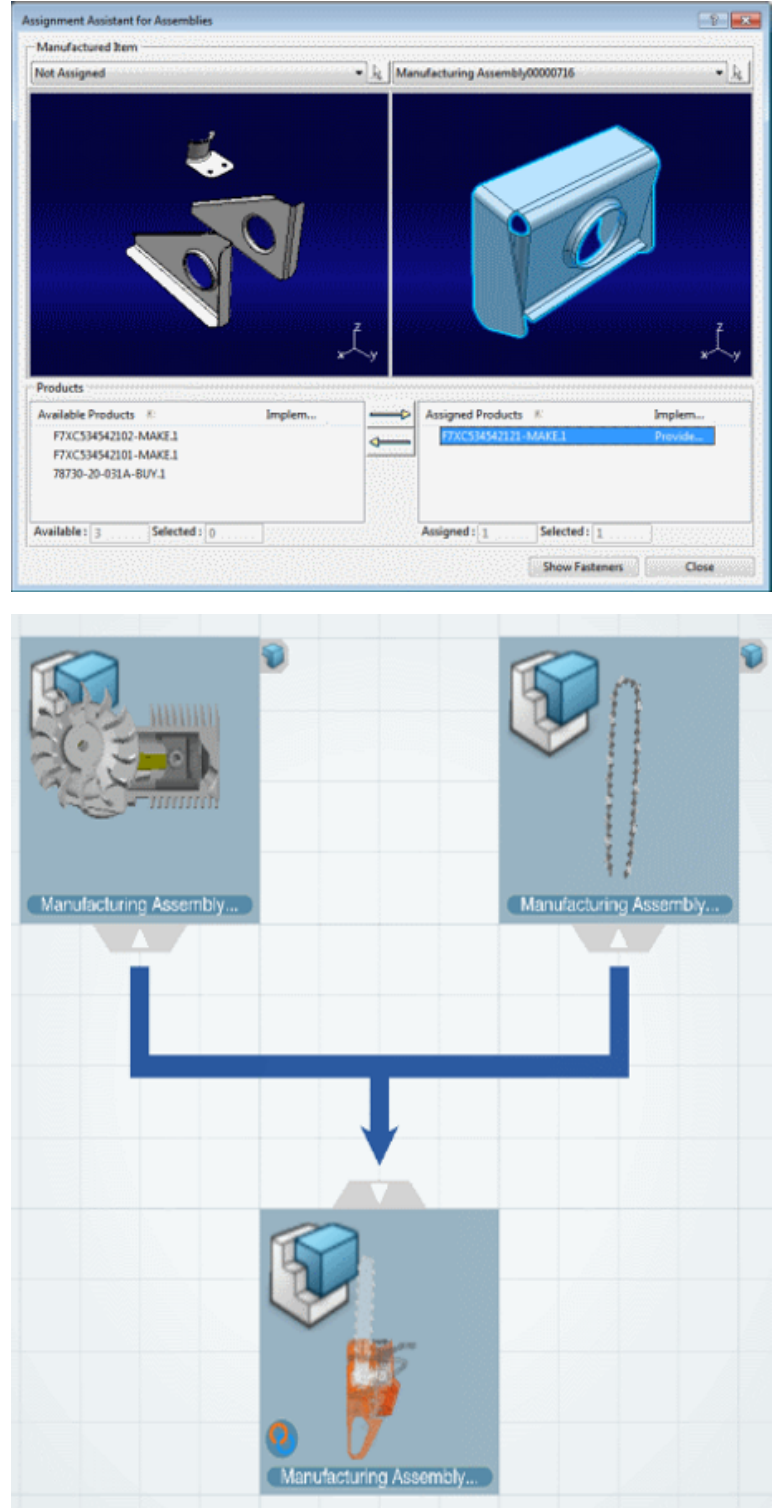

(3) Detailed design of assembly process: The distribution of components to the assembly process, you can directly select the desired part in the graphical interface assigned to the corresponding node, or from the product tree on parts assigned to the node, the system will automatically identify the distribution status of the parts, so as to avoid leakage of parts caused by process errors.

(4) Verification and optimization of assembly simulation: In the three-dimensional simulation environment including product, tooling, plant layout and human body model, the assembly process simulation is verified, the assembly process design errors are modified, the content of detailed process design is optimized, and finally the validated process design results are obtained.

The above proposed method was validated in the design process of a certain type of aircraft assembly process. According to the existing assembly process flow, all the assembled materials of the aircraft cockpit were programmed and simulated in sequence, and 12 problems of unreasonable process design and 6 problems of design
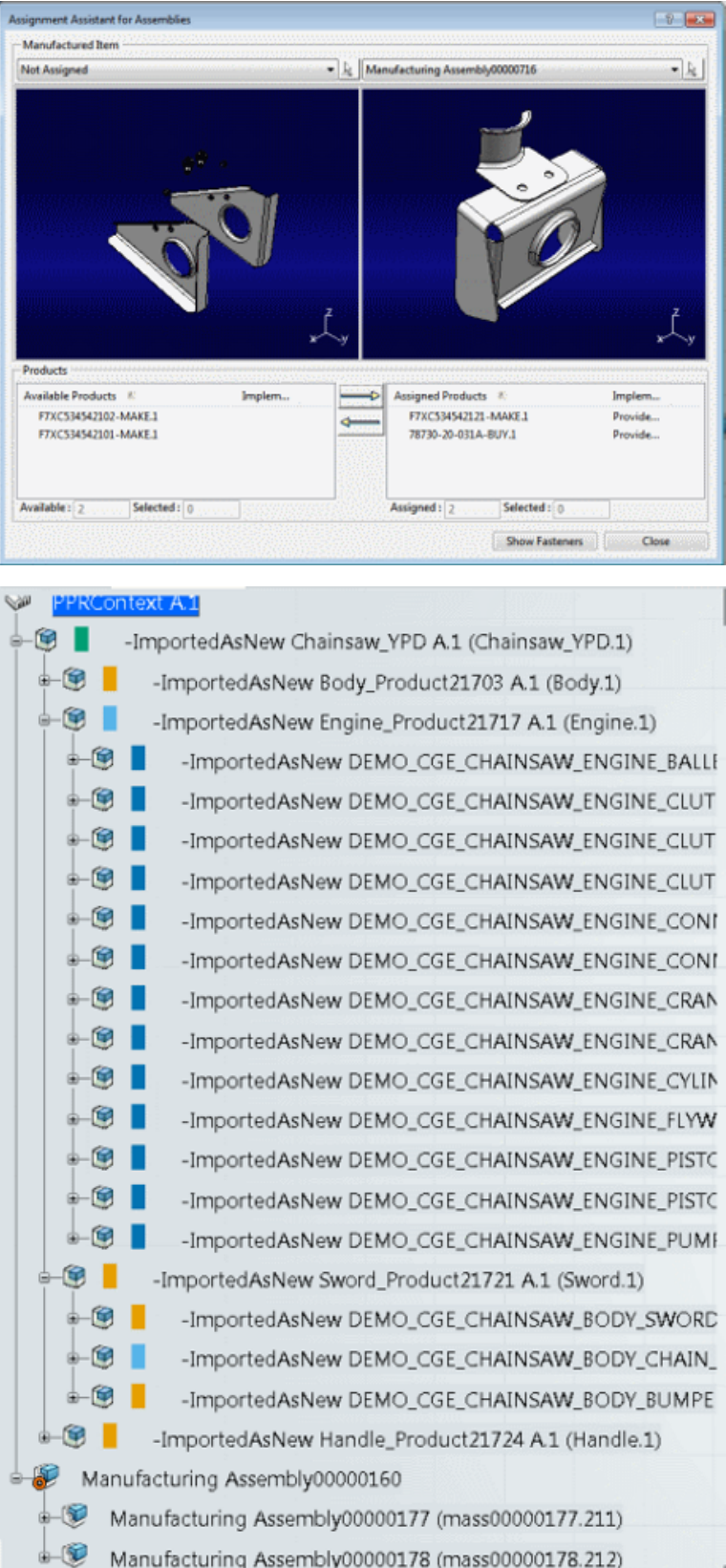

¿-(9) Manufacturing Assemblv00000178 (mass00000178.212)

of the interference were found. Through the $3 \mathrm{DE}$ platform, the problem of unreasonable process is optimized, which ensures the rationality of assembly sequence. At the same time, the design problems are returned to design changes, the design model is optimized, and the possible problems in assembly process are solved in advance.

\section{Summary}

This article presented a method to design aircraft digital assembly process based on 3D. The research showed that digital pre-assembly process can evaluate flaws in design prior to manufacturing. The assembly process design based on 3D model can improve the cooperativity of process design, reduce process planning problems caused by human factors, and break the traditional $2 \mathrm{D}$ drawing information serial transmission mode, realizes the 
collaborative assembly process planning and verification of aircraft tooling design.

\section{References}

1. Zhu W. Han H. Fang M. et al. Studies on visual scene process system of aircraft assembly[J]. Journal of Manufacturing Systems, 2013, 32(4):580-597.

2. Wu D W. Liang P. Research and Application of the Visual Assembly System Based on the Process Lightweight Model[J]. Advanced Materials Research, 2013, 655-657:1687-1690.

3. Yang J, Kim E, Hur M, et al. Knowledge Extraction and Visualization of Digital Design Process[J]. Expert Systems with Applications, 2017.

4. Li T. Lockett $H$. An Investigation into the Interrelationship between Aircraft Systems and Final Assembly Process Design [J]. Procedia Cirp, 2017, 60:62-67.

5. Sierla S, Kyrki V, Aarnio P, et al. Automatic assembly planning based on digital product descriptions[J]. Computers in Industry, 2018, 97:3446.

6. Ortegón J. Mas F. Gomez-Parra A. et al. Virtual improvement of a historical aircraft assembly line[J]. Manufacturing Engineering Society International Conference 2017, Vigo (Pontevedra), Spain 2017, 13:1312-1319.
7. Mingxing B. 3D planning and visual production based on $\mathrm{MBD}[\mathrm{J}]$. Aeronautical Manufacturing Technology, 2013(8): 40-43.

8. Gang S. Bile W. Jianhua L. et al. Secondary planet assembly process design and applied technology based on 3D model [J]. Computer Integrated Manufacturing Systems, 2011, 17(11):2343-2350.

9. Hongjie G. Ziming F. Yongliang Z. Xinghua Q. Design on Aircraft Smart Assembly Based Numerical Model[J]. Aeronautical Manufacturing Technology, 2017(11): 64-68.

10. Gómez A, Ríos J, Mas F, et al. Method and software application to assist in the conceptual design of aircraft final assembly lines[J]. Journal of Manufacturing Systems, 2016, 40:37-53.

11. FOURGEAU E. GOMEZ E. ADLI H. et al. System engineering workbench for multi-views systems methodology with 3DEXPERIENCE platform. The aircraft radar use case[M]//Complex Systems Design \& Management Asia. Berlin, Germany: Springer International Publishing, 2006. 